\title{
A Novel Filled Function Approach for Non-Smooth Global Optimization Problem
}

\author{
W.X. Wang \\ School of Science, Shanghai Second Polytechnic University \\ Shanghai, China
}

\author{
Y.L. Shang* \\ Department of Mathematics \\ Henan University of Science and Technology \\ Luoyang, China \\ *Corresponding author
}

\begin{abstract}
This paper presents a novel filled function approach for a general non-smooth box constrained global optimization problem. The idea of the filled function approach is that by utilizing a transforming function constructed at the given local minimizer of the objective function, the original problem could escape from the current local minimizer and identify an improved one. The proposed filled function contains two parameters, which can be readily adjusted at each iteration. The properties of the filled function are discussed, and a corresponding filled function algorithm is designed. Numerical experiments on several testing problems are implemented, and the preliminary computational results are also reported.
\end{abstract}

Keywords-non-smooth box constrained global optimization; filled function; filled function approach; global minimizer

\section{INTRODUCTION}

A variety of transforming function approaches have been developed to solve box constrained smooth global optimization problem $(P)$ : $\min _{x \in X} f(x)$, where $X$ is a box set. By applying any local search procedure to the proposed transforming function, the original problem is allowed to leave from one local minimizer and to identify a better one. This kind of methods include TRUST, the filled function method and the tunnelling method. In this paper, we focus on the filled function method. The filled function method was proposed initially by $\mathrm{Ge}[1]$ for continuous smooth global optimization problem $(P)$. The idea behind the filled functions is to construct an auxiliary function that allows us to escape from a given local minimum $x^{*}$. The filled function method contains two phases. In phases 1 , the problem $(P)$ searches for one of its local minimizer by using any extant local minimization procedure. When the phase 1 finished, the filled function method switches to phase 2. Phase 2 constructs a filled function and then minimize it to obtain an initial point for phase 1 . The aforementioned process is repeated until the original problem could never find its better minimizer. Later, the filled function was reconsidered by $[2,3,4,5]$. Note that all the above mentioned filled functions require the objective function to be smooth. In practice, however, the smoothness assumption is not always satisfied. In this paper, we extend the filled function method to include non-smooth case and construct a new filled function. The new filled function contains two parameters which can be easily adjusted at each iteration.

This paper is organized as follows: In Section 2, we make some assumptions on our problem and give the definition of filled function. In Section 3, we construct a novel filled function and discuss its properties. In Section 4, we state our algorithm based on the proposed filled function. In Section 5, we make a numerical test. Last, in Section 6, we give our conclusion.

\section{ASSUMPTIONS AND DEFINITION}

In this section, we first make some assumptions on the objective function and then define a filled function for nonsmooth global optimization.

To introduce the concept of a filled function approach for non-smooth global optimization, we need the following assumptions:

Assumption 1.The function $f(x)$ is Lipschitz continuous on $X$ with a $\operatorname{rank} L>0$.

Assumption 2.The problem $(P)$ has at least one global minimizer and has a finite number of different minimal function values.

The main tool used in the non-smooth filled function method is Clark generalized gradient. For the details about the Clark generalized gradient and its properties, the readers may refer to the literature [8].

Definition. A function $P\left(x, x^{*}\right)$ is called a filled function of $f(x)$ at a local minimizer $x^{*}$, if it has the following properties:

(I) $x^{*}$ is a strictly maximizer of $P\left(x, x^{*}\right)$.

(II) For any $x \in S_{1}=\left\{x \in X \backslash x^{*}: f(x) \geq f\left(x^{*}\right)\right\}$, one has $0 \notin \partial P\left(x, x^{*}\right)$.

(III) If $x^{*}$ is not a global minimizer of $f(x)$, then $P\left(x, x^{*}\right)$ has at least one minimizer in the set $S_{2}=\left\{x \in X: f(x)<f\left(x^{*}\right)\right\}$. 


\section{A NEW FILLED FUNCTION AND ITS PROPERTIES}

In this section, we will present a filled function. Let $\eta(t)$ be a function which has the following properties: $\eta(0)=0$ and $\eta^{\prime}(\mathrm{t}) \geq a>0$ for all $t>0$. Let $\varphi(t)$ be a function which meets the conditions below:

(1) $\varphi^{\prime}(t)>0$; (2) $\varphi^{\prime}(t)$ and $t \varphi^{\prime}(t)$ are decreasing monotonically to 0 ; (3) $\varphi(0)=0, \lim _{t \rightarrow+\infty} \varphi(t)=b>0$.

Denote $L(P)$ the set of the minimizers of the problem $(P)$. Let $X^{*} \in L(P)$, and define

$$
P\left(x, x^{*}, q, r\right)=\eta\left[\left(1+\left\|x-x^{*}\right\|\right)^{-1}\right] \varphi\left[q\left(f(x)-f\left(x^{*}\right)+r\right)\right]
$$

where $q>0$ and $r>0$ are two parameters. Let $D=\max _{x_{1}, x_{2} \in X}\left\|x_{1}-x_{2}\right\|$.

In the following, we will prove $P\left(x, x^{*}, q, r\right)$ is a filled function.

Lemma 1. Let $N\left(x^{*}\right)$ be a neighborhood of $x^{*}$ with the property that $f(x) \geq f\left(x^{*}\right)$ for all $x \in N\left(x^{*}\right) \bigcap X$, and $x_{t}=x^{*}+t\left(x-x^{*}\right) \quad$ with $\quad t \in(0,1) . \quad$ For $\xi_{t} \in \partial P\left(x_{t}, x^{*}, q, r\right)$, if $q>0$ is big enough such that $q \varphi^{\prime}(q r)<\frac{a}{L(1+D)^{2} \eta(1)} \varphi(q r)$, then one has $\xi_{t}^{T}\left(x-x^{*}\right)<0$.

Proof. Denote $f\left(x_{t}\right)-f\left(x^{*}\right)+r$ by $\mathrm{F}\left(x_{t}, \mathrm{r}\right)$. By the conditions and the fact that $\frac{x_{t}-x^{*}}{\left\|x_{t}-x^{*}\right\|}=\frac{x-x^{*}}{\left\|x-x^{*}\right\|}$, we have

$$
\begin{gathered}
\partial P\left(x_{t}, x^{*}, q, r\right) \subset-\eta^{\prime}\left(\frac{1}{1+\left\|x_{t}-x^{*}\right\|}\right) \frac{x-x^{*}}{\left(1+\left\|x_{t}-x^{*}\right\|\right)^{2}\left\|x-x^{*}\right\|} \times \\
\varphi\left[q F\left(x_{t}, \mathrm{r}\right)\right]+q \eta\left(\frac{1}{1+\left\|x_{t}-x^{*}\right\|}\right) \varphi^{\prime}\left[q F\left(x_{t}, \mathrm{r}\right)\right] \partial f\left(x_{t}\right)
\end{gathered}
$$

Thus, for any $\xi_{t} \in \partial P\left(x_{t}, x^{*}, q, r\right)$, there exists a point $z_{t} \in \partial f\left(x_{t}\right)$ such that

$$
\begin{gathered}
\xi_{t}=-\eta^{\prime}\left(\frac{1}{1+\left\|x_{t}-x^{*}\right\|}\right) \frac{x-x^{*}}{\left(1+\left\|x_{t}-x^{*}\right\|\right)^{2}\left\|x-x^{*}\right\|} \varphi\left[q F\left(x_{t}, \mathrm{r}\right)\right] \\
+q \eta\left(\frac{1}{1+\left\|x_{t}-x^{*}\right\|}\right) \varphi^{\prime}\left[q F\left(x_{t}, \mathrm{r}\right)\right] z_{t}
\end{gathered}
$$

By the properties of both $\eta(t)$ and $\varphi(t)$, it follows that

$$
-\eta^{\prime}\left(\frac{1}{1+\left\|x_{t}-x^{*}\right\|}\right) \frac{\left\|x-x^{*}\right\|}{\left(1+\left\|x_{t}-x^{*}\right\|\right)^{2}} \varphi\left[q F\left(x_{t}, \mathrm{r}\right)\right] \leq-\frac{a \varphi(q r)}{(1+D)^{2}}\left\|x-x^{*}\right\|
$$

$$
q \eta\left(\frac{1}{1+\left\|x_{t}-x^{*}\right\|}\right) \varphi^{\prime}\left[q F\left(x_{t}, \mathrm{r}\right)\right]\left(x-x^{*}\right)^{T} z_{t} \leq q L \eta(1) \varphi^{\prime}(q r)\left\|x-x^{*}\right\|
$$

By combining the above relations and performing simple calculations, we obtain

$$
\left(x-x^{*}\right)^{T} \xi_{t} \leq\left(q L \eta(1) \varphi^{\prime}(q r)-\frac{a \varphi(q r)}{(1+D)^{2}}\right)\left\|x-x^{*}\right\|
$$

Thus, if $q>0$ is big enough such that $q \varphi^{\prime}(q r)<\frac{a}{L(1+D)^{2} \eta(1)} \varphi(q r)$, then one has $\xi_{t}^{T}\left(x-x^{*}\right)<0$.

Theorem 1. If $q>0$ satisfies the condition of Lemma 1, then $x^{*}$ is a strict local

maximize $\mathrm{r}$ of $P\left(x, x^{*}, q, r\right)$.

Proof. Since $x^{*} \in L(P)$, there exists a neighborhood $N\left(x^{*}\right)$ of $x^{*}$ such that $f(x) \geq f\left(x^{*}\right)$ for all $x \in N\left(x^{*}\right) \cap \mathrm{X}$.

By the mean value theorem, there exist a point $x_{t}=x^{*}+t\left(x-x^{*}\right)$ and $\xi_{t} \in \partial P\left(x_{t}, x^{*}, q, r\right)$ with $t \in(0,1)$ such that

$$
P\left(x, x^{*}, q, r\right)-P\left(x^{*}, x^{*}, q, r\right)=\xi_{t}^{T}\left(x-x^{*}\right)
$$

By Lemma 1, if $q>0$ satisfies the condition of Lemma 1, then we have $\xi_{t}^{T}\left(x-x^{*}\right)<0$, which implies that $P\left(x, x^{*}, q, r\right)<P\left(x^{*}, x^{*}, q, r\right)$.

This shows that $x^{*}$ is a strict local maximize $r$ of $P\left(x, x^{*}, q, r\right)$.

Theorem 2. If $q>0$ satisfies the condition of Lemma 1, then for any $x \in S_{1}=\left\{x \in X \backslash x^{*}: f(x) \geq f\left(x^{*}\right)\right\}$, we have $0 \notin \partial P\left(x, x^{*}, q, r\right)$

Proof. Since $f(x) \geq f\left(x^{*}\right)$ and $x \neq x^{*}$, similar to the proof of Lemma 1, if $q>0$ satisfies the condition of Lemma 1,then we have $\xi^{T}\left(x-x^{*}\right)<0$, for all $\xi \in \partial P\left(x, x^{*}, q, r\right)$. Hence, we have $0 \notin \partial P\left(x, x^{*}, q, r\right)$

Theorem 3. Assume that $x^{*}$ is not a global minimizer. If $r$ satisfies $0<r<f\left(x^{*}\right)-f\left(x_{g}\right)$, where $x_{g}$ is a global minimizer of $(P)$, then there exists one point $x_{0} \in S_{2}=\left\{x \in X: f(x)<f\left(x^{*}\right)\right\}$ for which $x_{0}$ is a minimizer of $P\left(x, x^{*}, q, r\right)$. 
Proof. By the condition, we have $q\left(f\left(x_{g}\right)-f\left(x^{*}\right)+r\right)<0$, which implies that $P\left(x_{g}, x^{*}, q, r\right)<0$. Let $x_{0}$ be a global minimizer of $P\left(x, x^{*}, q, r\right)$ over

$X$, then it satisfies that $P\left(x_{0}, x^{*}, q, r\right) \leq P\left(x_{g}, x^{*}, q, r\right)<0$.

On the other hand, for any $x \in S_{1}$, we have $P\left(x, x^{*}, q, r\right)>0$. Thus, $x_{0} \in S_{2}$.

\section{SOLUTION ALGORITHM}

Based on the theoretic results in the previous section, the filled function method for $(P)$ is described as follows.

Filled function algorithm:

Initialization step

Let $q_{u}$ be the upper bound of parameter $q, r_{l}$ the lower bound of parameter $r, x_{1}$ the initial point and $e_{1}, e_{2}, \ldots, e_{2 n}$ the positive and negative coordinate directions. Set $k=1$, and go to the main step.

\section{Main step}

1.Starting from $x_{1}$, activate any non-smooth local minimization procedure to minimize $(P)$. Find the local minimizer $x_{1}^{*}$ and go to 2 .

2. Set $q=1$ and $r=1$.

3.Construct a filled function $P\left(x, x_{1}^{*}, q, r\right)$ and go to 3 .

4. If $k>2 n$, then go to 7 ; otherwise, set $x=x_{1}^{*}+0.1 e_{k}$, and take $x$ as an initial point to find a local minimizer of $x_{k}$ the problem:

$\min _{y \in X} P\left(y, x_{1}^{*}, q, r\right)$.

5. If $x_{k} \notin X$, then set $k=k+1$, and go to 4 ; otherwise, go to 6 .

6. If $f\left(x_{k}\right)<f\left(x_{1}^{*}\right)$, then (I) set $x=x_{k}, k=1$. (II) Using $x$ as a new initial point, apply any non-smooth local minimization procedure on problem $(P)$ to identify its another local minimizer $x_{2}^{*}$ with $f\left(x_{2}^{*}\right)<f\left(x_{1}^{*}\right)$. (III) Set $x_{1}^{*}=x_{2}^{*}$ and go to 2 ; otherwise, go to 7 .

7. Reduce $r$ by setting $r=0.1 r$. If $r \geq r_{l}$, then set $k=1$, and go to 3 ; otherwise, go to 8 .

8. Increase $q$ by setting $q=10 q$. If $q \leq q_{u}$, then set $k=1$, and go to 3 ; otherwise, take $x_{1}^{*}$ as a global minimizer, and the algorithm stops.

\section{Remarks:}

(1) The proposed filled function approach can also be applied to smooth box constrained global optimization problem.

(2) There are two phases in the filled function approach: local minimization and filling. In the first phase, a local minimizer $x^{*}$ is found. Any non-smooth local optimization algorithms can be used for this propose, such as Hybrid Hooke and Jeeves-Direct Method for Non-smooth Optimization [7], Mesh Adaptive Direct Search Algorithms for Constrained Optimization [6], Bundle methods, Powell's method, etc. In particular, the Hybrid Hooke and Jeeves-Direct Method is more preferable to others, since it is guaranteed to find a local minimum of a non-smooth function subject to simple bounds. In the phase 2, the constructed filled function $P\left(x, x^{*}, q, r\right)$ is minimized. During the minimization, if a point $x_{k}$ is found such that $f\left(x_{k}\right)<f\left(x^{*}\right)$, then the filling phase stops and the algorithm returns to the phase of local minimization to find a better solution. The aforementioned process repeats until the global minimizer is identified.

\section{NUMERICAL EXPERIMENT}

The proposed method has been used to solve many examples. In this section, we only present the results obtained for 2 examples. The computation was performed by a set of Fortran 95 programs. The filled function used in the test is of the form

$$
P\left(x, x^{*}, q, r\right)=\left(1+\left\|x-x^{*}\right\|\right)^{-1} \arctan \left[q\left(f(x)-f\left(x^{*}\right)+r\right)\right] .
$$

Problem 1.

$$
\begin{aligned}
& f(x)=\max \left\{5 x_{1}+x_{2},-5 x_{1}+x_{2}, x_{1}^{2}+x_{2}^{2}+4 x_{2}\right\}, \\
& X=\left\{\left(x_{1}, x_{2}\right)^{T}:\left|x_{1}\right| \leq 4,\left|x_{2}\right| \leq 4\right\}
\end{aligned}
$$

The algorithm successfully found a global solution: $x^{*}=(0,-3)^{T}$ with $f\left(x^{*}\right)=-3$. Table 1 records the numerical results of Problem 1.

Problem 2.

$$
\begin{aligned}
& f(x)=\frac{\pi}{4}\left[10 \sin ^{2} \pi x_{1}+\sum_{k=1}^{3}\left(x_{k}-1\right)^{2}\left(1+10 \sin ^{2} \pi x_{k+1}\right)+\left(x_{4}-1\right)^{2}\right], \\
& X=\left\{\left(x_{1}, x_{2}, x_{3}, x_{4}\right)^{T}:\left|x_{k}\right| \leq 10, k=1,2,3,4\right\} .
\end{aligned}
$$

The algorithm successfully found its global solution: $x^{*}=(1,1,1,1)^{T}$ with $f\left(x^{*}\right)=0$. Table 2 records the numerical results of Problem 2.

The symbols used in the tables are given below:

$k$ :The iteration number in finding the $k$ th local minimizer.

$\mathrm{r}$ : The parameter to find the $k+1$ th local minimizer.

$x_{k}$ :The $k$ th initial point to find the $k$ th local minimizer.

$x_{k}^{*}$ : The $k$ th local minimizer. 
$f\left(x_{k}\right)$ : The function value of the $k$ th initial point.

$f\left(x_{k}^{*}\right)$ : The function value of the $k$ th local minimizer.

\section{CONCLUSIONS}

This paper developed a computational method based on a new filled function for both non-smooth and smooth box constrained global optimization. The proposed filled function contains two parameters which can be easily adjusted at each iteration. Moreover, we make a numerical test. From our numerical studies, we observe that the proposed filled function approach is promising.

\section{ACKNOWLEDGEMENTS}

This work was supported by the NNSF of China (Nos.11471102 and 11001248), the SEDF under Grant No. 12YZ178, the key discipline "Applied Mathematics" of Shanghai Second Polytechnic University (No.A30XK1322100).

\section{REFERENCES}

[1] R.P.Ge \& Y.F.Qin, A class of filled functions for finding a global minimizer of a function of several variables, Journal of Optimization Theory and Applications, 54(2), pp. 241-252, 1987.

[2] X.Liu, Finding global minima with a computable filled function, Computers and Operations Research, 31, pp. 61-80, 2004.

[3] L.S.Zhang, On the solving global optimization approach from local to global,Journal of Chongqing Normal University(Natural Science), 26(1), pp. 1-6, 2009.

[4] L.S.Zhang, C.K.Ng \& D.Li, A new filled function method for global optimization, Journal of Global Optimization, 28, pp. 17-43,2004.

[5] Y.J.Yang \& Y.L.Shang, A new filled function method for unconstrained global optimization, Applied Mathematics and Computation, 173, pp. 501-512, 2006.

[6] C. Audet \& J.E. Dennis Jr., Mesh adaptive direct search algorithms for constrained optimization, SIAM Journal on Optimization, 17, pp. 188217, 2006.

[7] C. J. Price, B. L. Robertson \& M. Reale., A hybrid Hooke and Jeeves direct method for non-smooth optimization, Advanced Modelling and Optimization, 11(1), pp. 43-61,2009.

[8] F.H.Clarke., Optimization and non-smooth analysis, New York: John Wiley and Sons, 1983.

TABLE I. COMPUTATIONAL RESULTS FOR PROBLEM 1

\begin{tabular}{|c|c|c|c|c|}
\hline$k$ & $r$ & $x_{k}$ & $x_{k}^{*}$ & $f\left(x_{k}^{*}\right)$ \\
\hline 1 & - & $\left(\begin{array}{l}1 \\
1\end{array}\right)$ & $\left(\begin{array}{l}0.0000 \\
0.0000\end{array}\right)$ & 0.0000 \\
\hline 2 & 0.1 & $\left(\begin{array}{l}-0.0002 \\
-0.9725\end{array}\right)$ & $\left(\begin{array}{l}-0.0002 \\
-0.9725\end{array}\right)$ & -0.9715 \\
\hline 3 & 0.1 & $\left(\begin{array}{l}-0.0003 \\
-2.5644\end{array}\right)$ & $\left(\begin{array}{l}-0.0000 \\
-3.0000\end{array}\right)$ & -3.0000 \\
\hline
\end{tabular}

TABLE II. COMPUTATIONAL RESULTS FOR PROBLEM 2.

\begin{tabular}{|c|c|c|c|c|}
\hline$k$ & $r$ & $x_{k}$ & $x_{k}^{*}$ & $f\left(x_{k}^{*}\right)$ \\
\hline 1 & - & $\left(\begin{array}{l}2 \\
2 \\
2 \\
2\end{array}\right)$ & $\left(\begin{array}{l}1.9898 \\
1.9896 \\
1.9896 \\
1.9896\end{array}\right)$ & 3.1096 \\
\hline 2 & 0.1 & $\left(\begin{array}{l}1.0979 \\
1.9865 \\
1.9896 \\
1.9898\end{array}\right)$ & $\left(\begin{array}{l}1.0000 \\
1.0000 \\
1.0000 \\
1.0000\end{array}\right)$ & 0.0000 \\
\hline
\end{tabular}

